\title{
Technologies and Geriatric Surgery: In Specialty Centres with Patients at the Center
}

\author{
Silvestro Canonico* and Gianluca Pellino \\ Department of Medical, Surgical, Neurological, Metabolic and Ageing Sciences, Second University of Naples, Italy
}

Over the last decades life expectancy has been progressively increasing in Western Countries. Consequently, the rate of elderly patients needing medical and surgical treatment for chronic as well as for acute conditions is being shifted higher [1].

Surgery for frail and elderly patients has some peculiarities. These individuals often have co-morbidities [2], need to take one or more drugs at the time of surgery and may be temporarily or permanently unable to take care of themselves autonomously. The peri-operative period could hence be very demanding for these patients and their relatives. Every effort should be made to reduce operative time, perioperative complications and length of stay in the hospital.

Surgical principles remain the same, but it is important to involve elderly patients and who is taking care of them in surgical decision making. Patients are to be evaluated in the light of their disease stage but also - more importantly -of their performance status [2]. Geriatric surgeons must aim to radicality, to cure the disease for which surgery is being undertaken. At the same time it is fundamental to bear in mind that patients' nuisances and their will to trade on surgery (and its potential consequences) should always guide the surgeons dealing with the elderly population. Aiming to reduce complications and optimise treatment, a relevant contribution is given by recent technological advancements in surgery and pre-operative instrumental imaging [3].

It is now accepted that robotic and laparoscopic surgery [4-6] can allow for better structures visualization, reduce intraoperative blood loss, postoperative pain, and postoperative ileus, allowing earlier patients mobilization in colorectal surgery. Advantages are even greater following the enhanced recovery after surgery (ERAS) pattern $[7,8]$. However, to fully benefit from a mini-invasive approach with earlier return to everyday activities, laparoscopic surgery and ERAS in the elderly must be used by experienced laparoscopic surgeons and in Units in which the ERAS protocol is being performed routinely.

Vessel sealing devices are nowadays widely adopted also in the elderly. Bipolar radiofrequency-activated and ultrasound instruments are very useful in these patients, both in laparoscopic and in open surgery, allowing for minimized blood loss, precise tissues dissection and reduced transmission to surrounding structures $[9,10]$. Differently from conventional diathermy devices, these scalpels can also be safely adopted in patients with permanent pacemakers without provoking EKG disturbances [11]. There are specific diseases in which their use can offer advantages, such as thyroid surgery $[9,12]$. Elderly patients more often present with large goitres with compression and dislocation of adjacent structures which need to be identified and spared. Anaplastic carcinoma is a feature of older patients: it is highly invasive and easily bleeding, hence tissue sealing devices as well as instrumentation to identify nervous structures [13] can be extremely useful.

Proctologic diseases are another field of application of energy technologies. When dealing with elderly patients suffering from proctologic disturbances, the perineal approach is to be preferred. Radiofrequency allows for haemorrhoidectomy being performed also in ambulatory settings, under local anesthesia, eliminating the need of hospitalizations [10]. It is effective in reducing postoperative pain (and need for analgesic drugs intake) and bleeding [10,14]. Trans-anal stapled procedures, namely procedure for prolapsing haemorrhoids $(\mathrm{PPH})$ or stapled haemorrhoidopexy $(\mathrm{SH})$ and stapled trans-anal rectal resection (STARR), have gained wide popularity recently for the treatment of III-degree piles without external component and obstructed defecation syndrome [15]. These techniques are reported to result in lower postoperative pain $[15,16]$. However, this observation should be counterbalanced with the higher rate of recurrences in the long-term follow-up and with several serious complications (even life-threatening ones) which have been associated with the procedures [15-17]. Careful patient selection is necessary, not to overlook potential underlying diseases $[15,17,18]$. We recommend conventional treatments, implemented by a prudent use of the above described technologies - avoiding blind trans-anal stapling.

Ulcers, wounds which do not heal spontaneously, are often observed in elderly population. Regardless of the etiopathogenesis, a correct wound bed preparation for covering graft is paramount. High pressure fluid jet delivered by a hydrosurgery system offers better wound debridement than conventional methods without additional complications, and its use should therefore be encouraged in the elderly $[19,20]$. Negative pressure wound therapy (NPWT) has an established role in fixing dermal substitutes to acute as well as chronic wounds [21]. The fields of application of NPWT go far beyond: it is particularly useful in the eventuality of abdominal and perineal wound dehiscence, and in patients with entero-cutaneous fistulas. It is also suitable in wound breakdown in patients with a prosthetic mesh, allowing for mesh preservation and avoiding infections [22]. Side-effects include hemorrhage, protein and electrolytes loss and presumed reduced blood flow on the wall of bowel loops which are directly exposed to NPWT [23]. However, recent advances highlighted its safety in experienced hands. Newer devices are also less expensive, easier to manage and suitable for home-delivered treatment.

Elderly patients are also often suffering from groin hernia. Studies $[24,25]$ showed the usefulness of fibrin glue mesh fixing in reducing the blood loss, making it particularly suitable for patients with coagulation disorders [26]. Moreover, its beneficial effects are much more pronounced when newer large-pored light-weight meshes are used, achieving lower postoperative pain and less seroma formation [25].

Finally, palliation is another reason to advocate treatment of elderly patients. They may present with recurrent or advanced cancer lesions or may be unsuitable for extended procedures. Skin lesions due

*Corresponding author: Silvestro Canonico, Director, Department of Medical Surgical, Neurological, Metabolic and Ageing, Sciences, Second University of Naples, Piazza Miraglia 5, 80138 Naples, Italy, Tel: + 39-81-5665278; Fax: + 39 81-5665278; E-mail: silvestro.canonico@unina2.it

Received May 13, 2013; Accepted May 14, 2013; Published May 16, 2013

Citation: Canonico S, Pellino G (2013) Technologies and Geriatric Surgery: In Specialty Centres with Patients at the Center. J Gerontol Geriat Res 2: e121. doi:10.4172/2167-7182.1000e121

Copyright: (c 2013 Canonico S, et al. This is an open-access article distributed under the terms of the Creative Commons Attribution License, which permits unrestricted use, distribution, and reproduction in any medium, provided the original author and source are credited. 
to breast or cutaneous cancers presenting with bleeding, malodorous discharge and pain may significantly impair social functioning and quality of life. In such frail patients electro-chemotherapy (ECT) ablation of lesions can be particularly favourable [27]. ECT delivers locally high concentrations of anti-neoplastic drugs using the principles of electroporation, applied by means of specific devices, significantly limiting systemic drug toxicity. It is a safe procedure, which requires short operative time (not over 30 minutes) and can be performed under sedation or local anesthesia (we favourite the former). We use ECT in secondary or recurrent skin lesions in elderly patients operated on for breast or skin cancers. We observed no ECT-related adverse events or complications, and all treated patients achieved a significantly higher quality of life with important remission or removal of nuisances due to cancer lesions, especially of pain [27].

Age should not be considered a reason to exclude patients from curative, advanced procedures [28]. Surgical decision making in elderly patients is complex, and results from a balance between patients' general health status, patients' wishes and expectations, disease stage and related disturbances, and surgeons' experience. Advanced and functional imaging techniques may allow for a pre-operative objective assessment, and are encouraged when indicated [3]; development of enhanced pre-operative assessment scores is desirable [2]. Technology is certainly useful in assisting correct operative techniques. However, as in general population, technology will surely not amend a "wrong incision". Technological advancements in geriatric surgery are to be used by experienced surgeons and on condition that no imbalance is produced in the correct process to pose indications to surgery; otherwise, frail patients will be likely experiencing complications leading to worse outcomes and - most importantly - worse quality of life.

\section{References}

1. Etzioni DA, Liu JH, Maggard MA, Ko CY (2003) The aging population and its impact on the surgery workforce. Ann Surg 238: 170-177.

2. Béland SG, Tannenbaum C, Ducruet T, Préville M, Moride Y (2013) Development of a Comorbidity Score Based on Institutionalization: Comparative Performance with a Comorbidity Score Modeled on Mortality. J Gerontol Geriat Res 2: 116.

3. Selvaggi F, Cuocolo A, Sciaudone G, Maurea S, Giuliani A, et al. (2003) FGDPET in the follow-up of recurrent colorectal cancer. Colorectal Dis 5: 496-500.

4. Sun J, Li J, Wang J, Pan T, Zhou J, et al. (2012) Meta-analysis of randomized controlled trials on laparoscopic gastrectomy vs. open gastrectomy for distal gastric cancer. Hepatogastroenterology 59: 1699-1705.

5. Gonzalez R, Smith CD, Mattar SG, Venkatesh KR, Mason E, et al. (2004) Laparoscopic vs open resection for the treatment of diverticular disease. Surg Endosc 18: 276-280

6. Guillotreau J, Miocinovic R, Gamé X, Forest S, Malavaud B, et al. (2012) Outcomes of laparoscopic and robotic radical cystectomy in the elderly patients. Urology 79: 585-590.

7. Gravante G, Elmussareh M (2012) Enhanced recovery for colorectal surgery: Practical hints, results and future challenges. World J Gastrointest Surg 4: 190198

8. Lemanu DP, Singh PP, Berridge K, Burr M, Birch C, et al. (2013) Randomized clinical trial of enhanced recovery versus standard care after laparoscopic sleeve gastrectomy. Br J Surg 100: 482-489.

9. Dhepnorrarat RC, Witterick IJ (2013) New technologies in thyroid cancer surgery. Oral Oncol doi: 10.1016/j.oraloncology.2013.03.445.
10. Selvaggi F, Sciaudone G, Giuliani A (2005) New ambulatory treatment with radiofrequency for internal symptomatic hemorrhoids: morbidity evaluation. Gastroenterol Clin Biol 29: 939-940.

11. Nandalan SP, Vanner RG (2004) Use of the harmonic scalpel in a patient with a permanent pacemaker. Anaesthesia 59: 621

12. Parmeggiani $U$, Avenia $N$, De Falco $M$, Parmeggiani $D$, Pisaniello $D$, et al. (2005) Major complications in thyroid surgery: utility of bipolar vessel sealing (Ligasure Precise). G Chir 26: 387-394.

13. Parmeggiani D, De Falco M, Avenia N, Sanguinetti A, Fiore A, et al. (2012) NIM vs Neurosign in nerve sparing total thyroidectomy. Multicentric experience. Ann Ital Chir 83: 233-238.

14. Milito G, Cadeddu F, Muzi MG, Nigro C, Farinon AM (2010) Haemorrhoidectomy with Ligasure vs conventional excisional techniques: meta-analysis of randomized controlled trials. Colorectal Dis 12: 85-93.

15. Bove A, Bellini M, Battaglia E, Bocchini R, Gambaccini D, etal. (2012) Consensus statement AIGO/SICCR diagnosis and treatment of chronic constipation and obstructed defecation (part II: treatment). World J Gastroenterol 18: 4994 5013.

16. Sciaudone G, Di Stazio C, Guadagni I, Selvaggi F (2008) Rectal diverticulum: a new complication of STARR procedure for obstructed defecation. Tech Coloproctol 12: 61-63.

17. Basso L, Pescatori M, La Torre F, Destefano I, Pulvirenti D'Urso A, et al (2013) Emerging technologies in coloproctology: results of the Italian Society of Colorectal Surgery Logbook of Adverse Events. Tech Coloproctol 17: 207-211.

18. Selvaggi F, Sciaudone G, Guadagni I, Pellino G (2010) Ileal pouch-anal anastomosis after stapled haemorrhoidopexy for unrecognized ulcerative colitis. Colorectal Dis 12: e172.

19. Canonico S, Campitiello F, Della Corte A, Padovano V, Pellino G (2013) Treatment of leg chronic wounds with dermal substitutes and thin skin grafts, InTech, doi: 10.5772/51852.

20. Gravante G, Delogu D, Esposito G, Montone A (2007) Versajet hydrosurgery versus classic escharectomy for burn débridment: a prospective randomized trial. J Burn Care Res 28: 720-724.

21. Sorrentino VP, Corte AD, Fattopace A, Campitiello F, Ferretti M, Canonico $S$ (2011) The use of negative pressure wound therapy (npwt) and dermal substitutes in the treatment of diabetic foot ulcers. BMC Geriatrics 11

22. Baharestani MM, Gabriel A (2011) Use of negative pressure wound therapy in the management of infected abdominal wounds containing mesh: an analysis of outcomes. Int Wound J 8: 118-125

23. Lindstedt S, Malmsjö M, Hansson J, Hlebowicz J, Ingemansson R (2012) Microvascular blood flow changes in the small intestinal wall during conventional negative pressure wound therapy and negative pressure wound therapy using a protective disc over the intestines in laparostomy. Ann Surg 255:171-175.

24. Negro P, Basile F, Brescia A, Buonanno GM, Campanelli G, et al. (2011) Open tension-free Lichtenstein repair of inguinal hernia: use of fibrin glue versus sutures for mesh fixation. Hernia 15: 7-14.

25. Canonico S, Benevento R, Perna G, Guerniero R, Sciaudone G, et al. (2013) Sutureless fixation with fibrin glue of lightweight mesh in open inguinal hernia repair: effect on postoperative pain: a double-blind, randomized trial versus standard heavyweight mesh. Surgery 153: 126-130.

26. Canonico S, Sciaudone G, Pacifico F, Santoriello A (1999) Inguinal hernia repair in patients with coagulation problems: prevention of postoperative bleeding with human fibrin glue. Surgery $125: 315-317$.

27. Benevento R, Santoriello A, Perna G, Canonico S (2012) Electrochemotherapy of cutaneous metastastes from breast cancer in elderly patients: a preliminary report. BMC Surg 12: S6.

28. Petronella $P$, Scorzelli $M$, Manganiello A, Nunziata L, Ferretti $M$, et al. (2010) Our experience of total mesorectal excision for rectal cancers. Hepatogastroenterology 57: 482-486. 